\title{
Geociências
}

\section{Inclusões fluidas em topázio do Complexo Granítico Estanífero de Massangana (RO)}

\author{
Valmir da Silva Souza \\ Professor do Departamento de Geociências da Universidade do Amazonas \\ E-mail:valmirsouza@ufam.edu.br
}

Rodrigo Malheiros Pontes

Bolsista do Programa de Iniciação Científica do CNPq do Curso de Geologia da Universidade de Brasília

E-mail:hualalai@ig.com.br

Márcia Abrahão Moura

Professora do Instituto de Geociências da Universidade de Brasília

E-mail:mamoura@unb.br

\section{Resumo}

O complexo granítico de Massangana, localizado na região central de Rondônia, sudoeste do Cráton Amazônico, Brasil, é um importante exemplo da Província Estanífera de Rondônia. Esse complexo possui dimensões batolíticas, marcantes estruturas anelares relacionadas às sucessivas fases magmáticas e hospeda mineralizações de $\mathrm{Sn}, \mathrm{W}, \mathrm{Nb}$, Ta, topázio e berilo associadas a pegmatitos. Esse trabalho apresenta a composição química e as condições mínimas de aprisionamento dos fluidos nos cristais de topázio encontrados no complexo granítico estanífero de Massangana. O topázio, de cor azul, apresenta qualidade gemológica e possui cinco grupos de inclusões fluidas de natureza $\mathrm{H}_{2} \mathrm{O}-\mathrm{NaCl}$, cuja salinidade equivalente varia de 3,4 a $11,7 \%$ em peso de $\mathrm{NaCl}$, densidade em torno de $0,75 \mathrm{~g} / \mathrm{cm}^{3}$ e temperatura de homogeneização total (Th) entre 320 e $350^{\circ} \mathrm{C}$. Devido à íntima associação do topázio com cassiterita, wolframita e columbita-tantalita nos corpos pegmatíticos, admite-se que esse intervalo de Th também corresponda ao intervalo mínimo de cristalização desses outros minerais metálicos. Considerando que os pegmatitos no complexo granítico de Massangana estão associados às últimas fases magmáticas, é provável que a profundidade de posicionamento desses corpos pegmatíticos seja inferior a $3 \mathrm{~km}$ e a uma pressão estimada entre 1 e 1,5 kbar.

Palavras-chave: complexo granítico de Massangana, topázio gemológico, inclusões fluidas, Roraima.

\begin{abstract}
The Massangana granitic complex, located in the central region of the State of Rondonia, State, southwestern of the Amazon craton, Brazil, is an important example of the Rondônia Tin Province. The complex represents a batholitic body with conspiscuous ring structures related to successive intrusion events hosting $\mathrm{Sn}, \mathrm{W}, \mathrm{Nb}, \mathrm{Ta}$, topaz and beryl mineralizations associated to pegmatite. In this paper we determine the chemical composition and the minimal conditions of capture of the fluids in topaz from the Massangana granitic complex. The blue topaz has gemological quality and presents five groups of $\mathrm{H}_{2} \mathrm{O}-\mathrm{NaCl}$ fluid inclusions, with salinity between 3,4 to $11,7 \%$ weight $\% \mathrm{NaCl}$ equivalent, density about $0,75 \mathrm{~g} / \mathrm{cm}^{3}$ and homogenization temperature between 320 to $350^{\circ} \mathrm{C}$. The close association of topaz with cassiterite, wolframite and columbite-tantalite in pegmatites suggests the same minimal homogenization temperature for the crystallization of the mentroned metalic minerals. The pegmatitic bodies in the Massangana Complex are associated with the last magmatic phases and their probable emplacement depth is less than $3 \mathrm{~km}$ corresponding to an estimated pressure between 1 to 1,5 kbar.
\end{abstract}

Keywords: Massangana granitic complex, gemological topaz, fluid inclusions, Roraima. 


\section{Introdução}

O complexo granítico estanífero de Massangana está localizado na região central de Rondônia, distante cerca de $220 \mathrm{~km}$ ao sul da capital Porto Velho (Figura 1). Essa região abriga os principais depósitos de estanho da Província Estanífera de Rondônia e foi alvo de intensa atividade de mineração por cerca de 40 anos (Dall'Igna, 1996). Atualmente, de- vido ao baixo preço do estanho no mercado internacional, apenas poucos garimperiros atuam na área do complexo granítico de Massangana, extraindo principalmente topázio e um pouco de cassiterita ao longo de pequenos aluviões. O topázio é comercializado como gema e seu jazimento primário está associado a corpos pegmatíticos, os quais foram, em grande parte, erodidos e transportados para os aluviões.
Esse trabalho objetiva caracterizar a composição química e as condições mínimas de aprisionamento dos fluidos nos cristais de topázio encontrados no complexo granítico estanífero de Massangana. A metodologia de trabalho envolveu estudos petrográfico e microtermométrico em quatro seções polidas em ambas as faces. No estudo microtermométrico utilizou-se a platina Chaixmeca modelo 871 do Laboratório de Inclusões

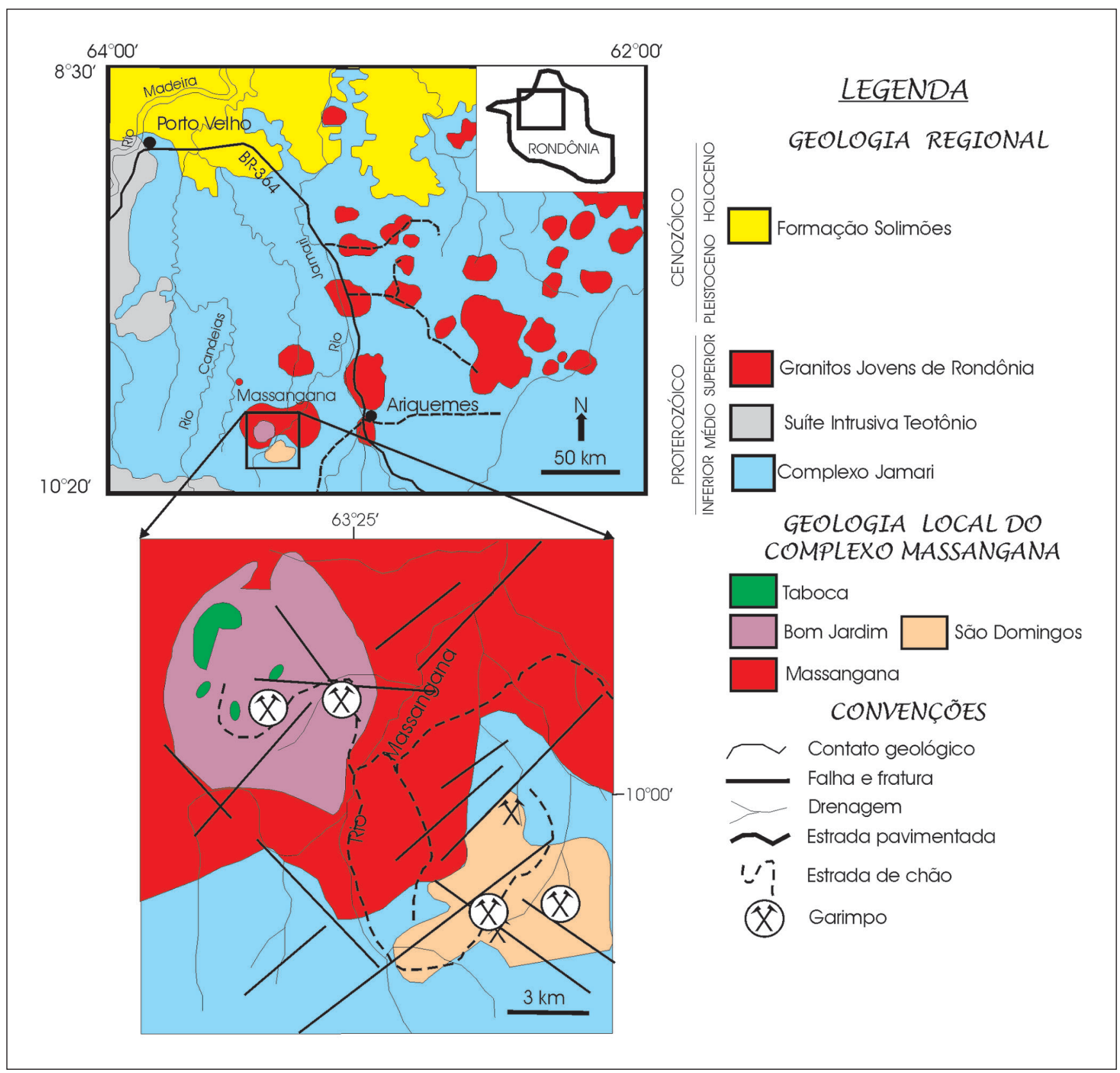

Figura 1 - Distribuição dos principais depósitos de estanho na Província Estanífera de Rondônia, destacando o mapa geológico do Complexo Granítico de Massangana (Bettencourt \& Dall'Agno, 1995). 
Valmir da Silva Souza et al.

Fluidas do Instituto de Geociências da Universidade de Brasília (IG-UnB). O equipamento foi calibrado utilizando-se inclusões fluidas sintéticas, com precisão variando de $\pm 1^{\circ} \mathrm{C}$ para resfriamento até $-100^{\circ} \mathrm{C}$ e de $\pm 5^{\circ} \mathrm{C}$ para aquecimento até $350^{\circ} \mathrm{C}$, com velocidade de aquecimento de cerca de $1^{\circ} \mathrm{C}$ por minuto. Duas inclusões fluidas foram ainda submetidas à análise por espectroscopia micro-Raman no Instituto de Física da Universidade Federal de Minas Gerais.

\section{Contexto geológico}

O complexo granítico de Massangana é um dos principais representantes dos chamados Granitos Jovens de Rondônia (Kloosterman, 1967 e 1968). Possui dimensões batolíticas e se destaca nas imagens de satélite e fotografias aéreas pelo seu formato elíptico e alongado na direção ESE-WNW, contendo estruturas anelares relacionadas às sucessivas fases magmáticas que constituem a história evolutiva desse batólito (Kloosterman, 1967 e 1968; Warghorn, 1974; Isotta et al., 1978). Romanini (1982) caracterizou quatro fases magmáticas, denominando-as de Massangana, Bom Jardim, São Domingos e Taboca (Figura 1). A fase Massangana é a principal e mais antiga, com idade U-Pb em zircão de 991_14Ma (Bettencourt et al., 1999), sendo constituída por um biotita granito cálcio-alcalino a alcalino, de cor rosa-claro, com textura porfirítica média a grossa e contendo megacristais de feldspato potássico. As fases Bom Jardim e São Domingos são intrusivas na fase Massangana, apresentam idade $\mathrm{Rb}$-Sr em rocha total da ordem de $956 \pm 9 \mathrm{Ma}$, com razão inicial $87 \mathrm{Sr} / 86 \mathrm{Sr}$ de $0,7104 \pm 0,0056$ (Priem et al., 1989), sendo representadas por dois stocks de biotita granito de composição alcalina, exibindo cor rosa-claro, textura inequigranular fina a média e contendo xenólitos de diferentes tipos de rochas. A fase Taboca é considerada mais jovem, pois é intrusiva na fase Bom Jardim e é representada por apófises sieníticos a quartzo-sieníticas, com textura inequigranular fina a média, também contendo xenólitas das rochas encaixantes.
Os depósitos de estanho no complexo granítico Massangana estão relacionados às fases Bom Jardim e São Domingos, com a cassiterita associada a veios de quartzo, greisens e corpos pegmatíticos, normalmente contendo wolframita, berilo, topázio e columbita-tantalita (Romanini, 1982; Bettencourt \& Dall'Agnol, 1995; Bettencourt et al., 1997). Os cristais de topázio comercializados como gemas ocorrem nas zonas intermediária e central dos corpos pegmatíticos heterogêneos, normalmente associados a berilo, cassiterita, wolframita e columbita-tantalita. Esses cristais de topázio são euédricos, de hábito prismático com terminações piramidais, com tamanho entre 3 e $12 \mathrm{~cm}$, são pouco fraturados, transparentes a translúcidos e apresentam cor variando de azul, amarelo a incolor.
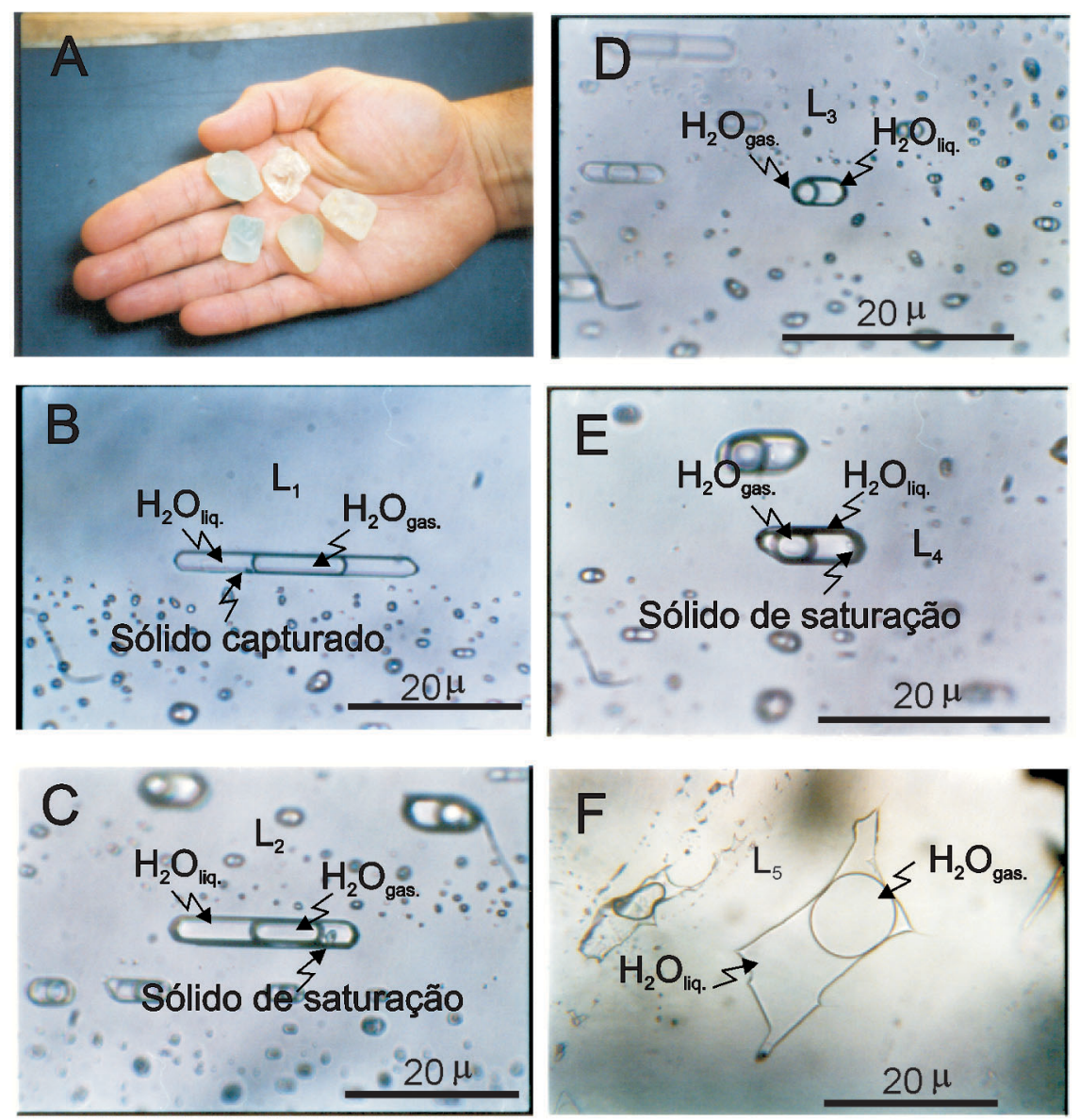

Figura 2 - Amostras de cristais de topázio gemológico (A) e características morfológicas das suas diferentes famílias de inclusões fluidas: família $L_{1}(B)$, família $L_{2}$ $(C)$, família $L_{3}(D)$, família $L 4(E)$ e família $L_{5}(F)$. 
grupos e/ou alinhados ao longo de planos de crescimento do cristal, enquanto que as inclusões fluidas pseudo-secundárias $\left(\mathrm{L}_{3}\right)$ tendem a ocorrer nas bordas dos cristais, distribuídas de modo aleatório.

O grupo $\mathrm{L}_{1}$ é do tipo bifásico, pois apresenta uma fase líquida e uma fase gasosa, exibe formato em bastonete com tamanho entre 20 e $40 \mu \mathrm{m}$, é transparente a levemente rosada, com contornos finos e o volume da fase gasosa (Vg) em relação à fase líquida variando de 40 a $50 \%$ (Figura 2B). Ocasionalmente, contém uma fase sólida de cor preta, hábito granular e tamanho inferior a $1 \mu \mathrm{m}$, a qual é interpretada como provável agregado mineral capturado durante a formação da cápsula hospedeira de fluidos e gases.

$\mathrm{O}$ grupo $\mathrm{L}_{2}$ é do tipo bifásico e é o mais frequiente. Apresenta formato elíptico a levemente prismático, ocasionalmente como cristal negativo, seu tama- nho varia de 2 a $8 \mu \mathrm{m}$, é transparente a translúcido, com contornos mais grossos e bem definidos, e Vg variando de 40 a $50 \%$ (Figura 2C).

O grupo $\mathrm{S}_{1}$ é raro, apresenta a mesma morfologia e $\mathrm{Vg}$ das inclusões fluidas do grupo $\mathrm{L}_{1}$, porém é constituído de inclusões fluidas trifásicas, pois apresentam uma terceira fase representada por um sólido de saturação incolor, de hábito cúbico e isótropo, interpretado como um sal do tipo halita, o qual ocupa um volume (Vs) em torno de $3 \%$ da inclusão fluida (Figura 2D).

$\mathrm{O}$ grupo $\mathrm{S}_{2}$ é do tipo trifásico e é o mais raro, apresenta formato elíptico a levemente prismático, tamanho entre $4 \mathrm{e}$ $8 \mu \mathrm{m}$, um Vg entre 50 e $60 \%$ e uma fase sólida de saturação com Vs inferior a $3 \%$ (Figura 2E).

O grupo $\mathrm{L}_{3}$ é constituído por inclusões fluidas bifásicas e é pouco freqüen- te. São consideradas como inclusões fluidas pseudo-secundárias por exibir formato irregular e tamanho variando de 40 a $90 \mu \mathrm{m}$. Essas inclusões fluidas são transparentes, com contornos finos e $\mathrm{Vg}$ entre 20 e $30 \%$ (Figura 2F).

A observação microtermométrica permitiu caracterizar esse sistema fluido como do tipo $\mathrm{H}_{2} \mathrm{O}-\mathrm{NaCl}$, o que foi comprovado por meio da espectroscopia micro-Raman (Figuras 3A e 3B). Durante o resfriamento das inclusões fluidas (até $-120^{\circ} \mathrm{C}$ ), o sistema fluido apresenta um intervalo de congelamento total entre -70 e $-80^{\circ} \mathrm{C}$. Já durante o processo de aquecimento (até $+360^{\circ} \mathrm{C}$ ), esse sistema fluido apresenta a primeira mudança de fase entre $-24,2^{\circ} \mathrm{e}-18,6^{\circ} \mathrm{C}$, correspondente à temperatura do eutético (Te), a qual é uma medida pouco precisa e muitas vezes de difícil observação. Segundo Shepherd et al. (1985), o intervalo de temperatura do eutético para o sistema flui-
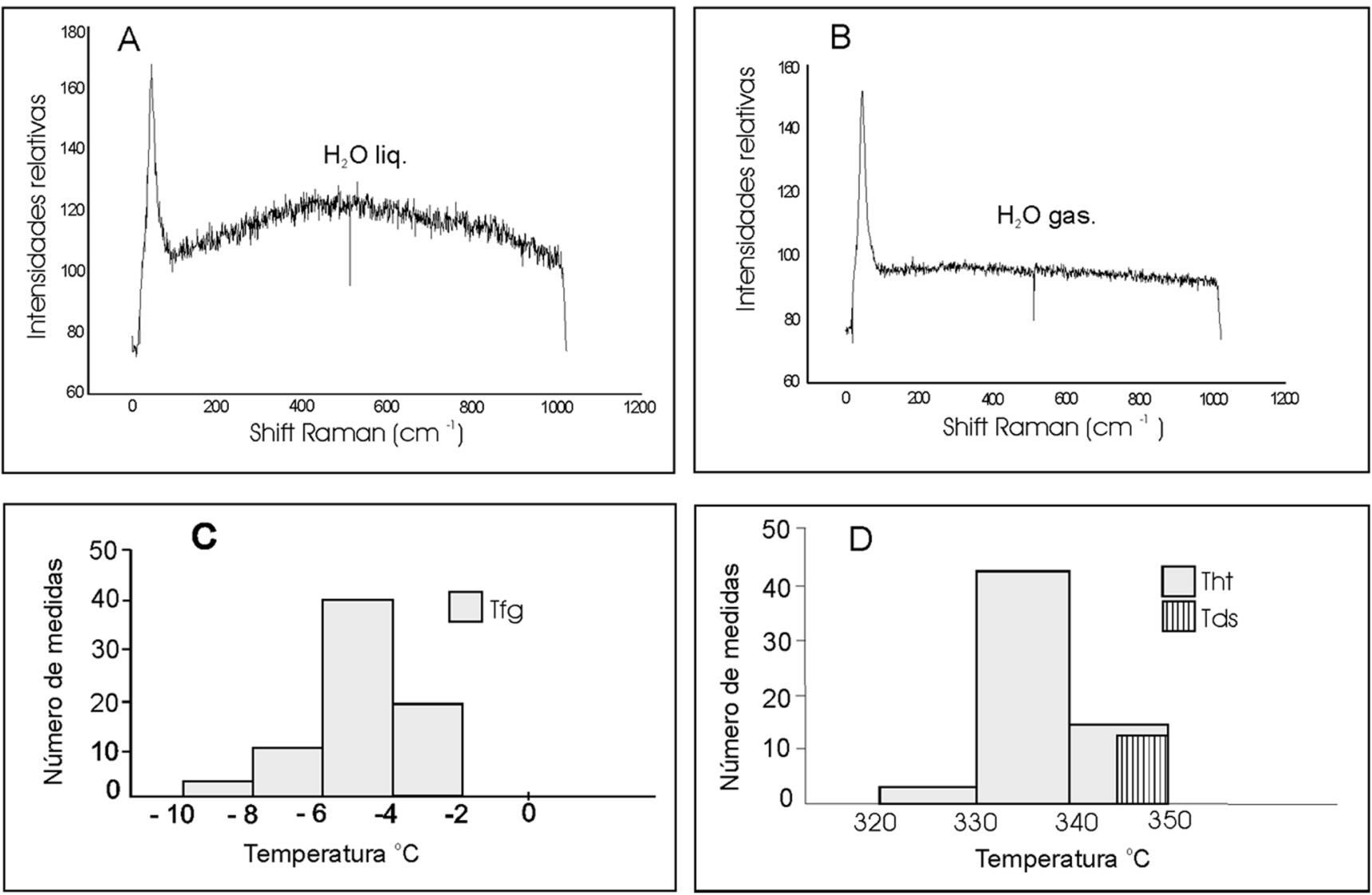

Figura 3 - Espectros de Raman para a fase aquosa (A) e gasosa (B) do sistema $\mathrm{H}_{2} \mathrm{O}-\mathrm{NaCl}-(\mathrm{KCL})$. Histograma com o intervalo de temperatura de fusão do gelo (C) e com o intervalo de tempertura de homogeneização total (D). 
Valmir da Silva Souza et al.

do $\mathrm{H}_{2} \mathrm{O}-\mathrm{NaCl}$ varia de $-21,2^{\circ}$ a $-20,8^{\circ} \mathrm{C}$. A segunda mudança de fase, correspondente à temperatura de fusão do gelo (Tfg), ocorre no intervalo de $-8^{\circ}$ a $-2^{\circ} \mathrm{C}$ (Figura 3C), sendo que para as inclusões fluidas $\mathrm{L}_{3}$ a Tfg $=-3,8^{\circ}$ a $-2^{\circ} \mathrm{C}$, para $\mathrm{L}_{1}$ a $\mathrm{Tfg}=-6,8$ a $-3,6^{\circ} \mathrm{C}$ e para $\mathrm{L}_{2}$ a $\mathrm{Tfg}=-8$ a $-5,3^{\circ} \mathrm{C}$. A temperatura de homogeneização total (Th) nas inclusões fluidas $\mathrm{L}_{1}$, $\mathrm{L}_{2}$ e $\mathrm{L}_{3}$ ocorre para a fase líquida no intervalo de 320 a $350^{\circ} \mathrm{C}$, porém com maior freqüência entre 330 e $340^{\circ} \mathrm{C}$ (Figura 3D). Nos casos das inclusões fluidas contendo sólidos de saturação $\left(\mathrm{S}_{1}\right.$ e $\left.\mathrm{S}_{2}\right)$, a fase sólida apresenta temperatura de dissolução (Tds) logo após a temperatura de homogeneização das fases líquida-gasosa $(\mathrm{L}+\mathrm{G}=\mathrm{L})$, ou seja, no intervalo entre 345 a $350^{\circ} \mathrm{C}$ (Figura 3D).

A salinidade do sistema $\mathrm{H}_{2} \mathrm{O}-\mathrm{NaCl}$, para as inclusões fluidas bifásicas $\left(\mathrm{L}_{1}, \mathrm{~L}_{2}\right.$ e $\mathrm{L}_{3}$ ), foi calculada com base na temperatura de fusão do gelo (Tfg), aplicandose a equação proposta por Bodnar (1993), onde se obteve um intervalo de salinidade equivalente de 3,4 a $11,7 \%$ em peso de $\mathrm{NaCl}$, distribuído no seguinte modo: inclusões fluidas $\mathrm{L}_{3}$ entre 3,4 e $6,1 \%, \mathrm{~L}_{1}$ entre 6 a 10,2\% e $L_{2}$ entre 8,3 e 11,7\% em peso de $\mathrm{NaCl}$ equivalente. Para as raras inclusões fluidas trifásicas $\left(S_{1}\right.$ e $\left.S_{2}\right)$, a salinidade foi calculada com base na temperatura de dissolução da fase sólida (Tds), aplicando-se a equação proposta por Sterner et al. (1988), onde se obteve uma salinidade equivalente entre $42 \mathrm{e}$ $42,5 \%$ em peso de $\mathrm{NaCl}$. A densidade das inclusões fluidas mais comuns $\left(\mathrm{L}_{1} \mathrm{e}\right.$ $\mathrm{L}_{2}$ ), estimada com base no diagrama que relaciona salinidade por temperatura de homogeneização total (Shepherd et al. 1985), apresenta um valor entre 0,65 e $0,75 \mathrm{~g} / \mathrm{cm}^{3}$.

\section{Considerações finais}

Em geral, nos corpos graníticos portadores de pegmatitos com mineralizações de Sn, W, Nb, e Ta e gemas, os sistemas fluidos são aquosos a aquocarbônicos, apresentam baixa a moderada salinidade, baixa densidade e temperatura mínima de aprisionamento entre
350 e $500^{\circ} \mathrm{C}$ (Campbell \& Panter, 1990 ; Linnen \& Williams-Jones, 1994; Lu \& Lottermoser, 1997). Estudos de inclusões fluidas em quartzo, topázio, fluorita e cassiterita nos sistemas de veios e greisens de outros depósitos de Sn e W na região central de Rondônia demonstram que a temperatura mínima de aprisionamento desses fluidos varia de 270 a $420^{\circ} \mathrm{C}$ (Souza \& Botelho, 2002; Leite Júnior, 2002).

No complexo granítico estanífero de Massangana, o sistema fluido no topázio, com características gemológicas, é de natureza essencialmente aquosa $\left(\mathrm{H}_{2} \mathrm{O}-\mathrm{NaCl}\right)$. Entretanto esse sistema apresenta salinidade variando de baixa a alta, ou seja, nas inclusões fluidas bifásicas $\left(\mathrm{L}_{1}, \mathrm{~L}_{2}, \mathrm{e} \mathrm{L}_{3}\right)$ a salinidade equivalente varia de 3,4 a $11,7 \%$ em peso de $\mathrm{NaCl}$, enquanto que nas raras inclusões fluidas trifásicas $\left(\mathrm{S}_{1}\right.$ e $\left.\mathrm{S}_{2}\right)$ a salinidade equivalente varia de 42 a $42,5 \%$ em peso de $\mathrm{NaCl}$. Essa variação no valor da salinidade é indicativa da provável mistura de fluidos de diferentes naturezas (por exemplo, magmático e meteórico) durante a ascensão do sistema fluido associado às fases Bom Jardim e São Domingos, modificando desse modo a salinidade dos fluidos mais primordiais, provavelmente representados pelas inclusões fluidas $\mathrm{S}_{1}$ e $\mathrm{S}_{2}$. Entretanto o modo de distribuição dos grupos de inclusões fluidas no interior dos cristais de topázio sugere uma variação nos valores de salinidade, de temperatura de homogeneização (Th) e de cronologia das inclusões fluidas, reflexo dos estágios progressivos de crescimento dos cristais. Nesse sentido, os primeiros grupos de inclusões fluidas formados apresentam os maiores valores de salinidade e Th $\left(\mathrm{L}_{1}, \mathrm{~L}_{2}, \mathrm{~S}_{1}\right.$ e $\left.\mathrm{S}_{2}\right)$ e ocupam as partes mais centrais dos cristais, ao contrário do grupo de inclusões fluidas pseudo-secundário $\left(\mathrm{L}_{3}\right)$ consideradas mais jovens e apresentando valores mais baixos de salinidade e Th. Todavia, o contraste entre os valores de Th para os grupos de inclusões fluidas primárias e pseudo-secundárias não é tão marcante, o que sugere que a temperatura no interior dos corpos pegmatíticos permaneceu elevada por um considerável período de tempo.
Com base nos critérios propostos por Van den Kerkhof e Hein (2001), esse sistema fluido apresenta característica de aprisionamento homogêneo, pois seu volume $\mathrm{Vg}$ varia muito pouco, tanto nas inclusões fluidas primárias, como nas pseudo-secundárias, o que demonstra também baixa variação na densidade desse fluido. Logo, a temperatura de homogeneização total aqui apresentada (Tht $=320$ e $350^{\circ} \mathrm{C}$ ) pode ser considerada como a temperatura mínima de aprisionamento desse fluido. Devido à íntima associação nos corpos pegmatíticos do topázio com cassiterita, wolframita e columbita-tantalita, admite-se que o intervalo de temperatura entre 320 e $350^{\circ} \mathrm{C}$ também corresponderia ao intervalo mínimo de cristalização desses outros minerais metálicos. Assim, é provável que o principal intervalo mínimo de temperatura de precipitação da cassiterita nos depósitos de estanho de Rondônia oscile na faixa de $300 \mathrm{a} 400^{\circ} \mathrm{C}$.

Os complexos graníticos com estruturas anelares são, normalmente, alojados em níveis crustais rasos e ao longo de falhamentos transcrustais (Johson et al., 2002). Segundo Okida (2001), os Granitos Jovens de Rondônia foram alojados em estruturas de alívio ao longo de linhas de fraquezas crustais, através da atuação de movimentos progressivos transpressivos-transtensivos. Considerando que os corpos pegmatíticos portadores de minerais de $\mathrm{Sn}, \mathrm{W}, \mathrm{Nb}$, e Ta e gemas no complexo granítico estanífero de Massangana estão associados às últimas fases magmáticas (Bom Jardim e São Domingos), é provável que a profundidade de posicionamento desses corpos seja inferior a $3 \mathrm{~km}$ e a uma pressão estimada na ordem de 1 a 1,5kbar.

\section{Agradecimentos}

À Companhia de Pesquisa e Recursos Minerais (CPRM-RO), através dos geólogos Rommel da Silva Sousa, Marcos Quadros, Jânio Leite de Amorim (in memorian) e Antônio Vieira, pelo apoio e discussões durante as atividades de campo. Ao Prof. Dr. Kazuo Fuzikawa (Laboratório de Inclusões Fluidas do CDTNCNEN) pelo apoio e discussões durantes as análises de micro-Raman. 


\section{Referências Bibliográficas}

BETTENCOURT, J.S., DALL'AGNOL, R. Symposium on rapakivi granites and related rocks. Excursion Guide: The rapakivi granites of the Rondonia Tin Province and associated mineralization. IGCP/Project 315, Belém, Brazil, 1995. 48p.

BETTENCOURT, J.S., LEITE Jr., W.B., PAYOLLA, B.L., SCANDOLARA, J.E., MUZZOLON, R., VIAN, J.A.J. The rapakivi granites of the Rondônia Tin Province, northern Brazil. In: INTERNATIONAL SYMPOSIUM ON GRANITES AND ASSOCIATED MINERALIZATIONS (ISGAM II). Salvador: Excursions Guide, 1997. 31p.

BETTENCOURT, J.S., TOSDAL, R.M., LEITE Jr., W.B., PAYOLLA, B.L. Mesoproterozoic rapakivi granites of the Rondônia Tin Province, southwestern border of the Amazonian craton, Brazil I. Reconnaissance U-Pb geochronology and regional implications. Precambrian Research, v.95, p.41-67,1999.

BODNAR, R.J. Revised equation and table for determination of the liquidus and isochores for a 40 wt. $\% \mathrm{H}_{2} \mathrm{O}-\mathrm{NaCl}$ solutions. Geochimica et Cosmochimica Acta, v.57, p.683-684, 1993.

CAMPBELL, A.R., PANTER, K.S. Coparation of fluid inclusions in coexisting (cogenetic?) wolframite, cassiterite, and quartz from St. Michael's Mountt and Cligga Head, Cornwall, England. Geochimica et Cosmochimica Acta, v.54, p.673-681, 1990.

DALL'IGNA, L.G. A mineração e o garimpo de cassiterita em Rondônia. A Terra em Revista. CPRM, v.1, p.56-61, 1996.

ISOTTA, C.A.L., CARNEIRO, J.M., KATO, H.T., BARROS, R.J.L. Projeto Província Estanífera de Rondônia. MME/DNPM/ CPRM, Porto Velho, v. 1, 407p., 1978. (Relatório Final).
JOHNSON, S.E., SCHIMIDT, K.L., TATE, M.C. Ring complexes in the Peninsular Tanges Batholith, Mexico and the USA: magma plumbing systems in the middle and upper crust. Lithos, v.61, p.187-208, 2002.

KLOOSTERMAN, J.B. Ring-structures in the Oriente e Massangana granite complexes, Rondônia, Brasil. Revista de Engenharia, Mineração e Metalurgia, v. 45, n.266, p.73-77, 1967.

KLOOSTERMAN, J.B. Uma província do tipo nigeriano no sul da Amazônia (Parte 1). Revista de Engenharia, Mineração e Metalurgia, v. 47, n.278,p.59-64, 1968.

LEITE JÚNIOR, W.B. A suite intrusiva Santa Clara (RO) e a mineralização primária polimetálica ( $\mathrm{Sn}, \mathrm{W}, \mathrm{Nb}, \mathrm{Ta}, \mathrm{Zn}, \mathrm{Cu}$ e Pb) associada. São Paulo: Instituto de Geociências, Universidade de São Paulo, 2002. 305p. (Tese de Doutorado).

LINNEN, R., WILLIAMS-JONES, A. The evolution of pegmatite-hosted Sn-W mineralization at Nong Sua, Thailand: Evidence from fluid inclusions and stable isotopoes. Geochimica et Cosmochimica Acta, v.58, n.2, p.735-747,1994.

LU J., LOTTERMOSER, B.G. Petrogenesis of rare-element pegmatites in the Olary Block, South Australia, part 2. Fluid inclusion study. Mineralogy and Petrology, v.59, p.21-41. 1997

OKIDA, R. Aplicação do sensoreamento remoto e aerogamaespectrometria ao estudo do controle estrutural dos granitos estaníferos de Rondônia. São Paulo: Instituto de Geociências, Universidade de São Paulo, 2001. 217p. (Tese de Doutorado).

PRIEM, H.N.A., BOM, E.H., VERDURMEN, E.A.T., BETTENCOURT, J.S. Rb-Sr chronology of Precambrian crustal evolution in Rondônia (western margin of the Amazonian craton), Brazil. Journal of South American Earth Sciences, v.2, n.2, p.163-170, 1989.
ROMANINI, S.J. Geologia e geoquímica do complexo granitóide de Massangana e sua relação com as mineralizações de estanho. Salvador: Curso de Pós-Graduação em Geociências da Universidade Federal da Bahia, 1982. 85p. (Dissertação de Mestrado).

SHEPHERD, T.J., RANKIN, A.H., ALDERTON, D.H.M. A practical guide to fluid inclusion studies. New York: Blackie \& Son Ltd., 1985, 239p.

SOUZA, V.S., BOTELHO, N.F. Geologia do depósito de estanho do Bom Futuro (Rondônia) e composição dos fluidos nos sistemas de veios e greisens. In: KLEIN, E. L., VASQUEZ, M. L., ROSA-COSTA, L. T. (Eds.). Contribuições à Geologia da Amazônia. Belém: SBG-Núcleo Norte, v.3, p.199-214, 2002.

STERNER, S.M., HALL, D.L., BODNAR, R.J. Synthetic fluid inclusions: V. solubility relations in the system $\mathrm{NaCl}-\mathrm{KCl}-\mathrm{H} 2 \mathrm{O}$ under vapor-saturated conditions. Geochimica et Cosmochimica Acta, v.52, p.989-1006, 1988.

VAN DEN KERKHOF, A.M., HEIN, U.F. Fluid inclusion petrography. In: ANDERSEN, T., FREZZOTTI, M. L., BURKE, E. A. J. (Ed.). Fluid Inclusions: Phase Relations Methods - Applications. Lithos (Special Volume), v.55, p.27-47, 2001.

WAGHORN, J.G. The geology of Rondônia, western Brazil, with special reference to the tin-bearing granite complexes and placer deposits. London: Faculty of Science, University of London, 1974. 293p., (Ph.D. Thesis).

\section{Artigo recebido em 10/04/2003 e aprovado em 02/12/2003.}

\section{6 - 2004}

REM - Revista Escola de Minas a mais antiga revista técnico-científica do setor mínero-metalúrgico: assine e publique 